\title{
EMuS in CSNS
}

\section{Guang Zhao'}

Institute of High Energy Physics

Beijing, China

E-mail: zhaog@ihep.ac.cn

In this presentation, we report the recent progress in EMuS at CSNS. The following topics will be discussed: a) the base design for neutrino mode, b) the optimized design for surface muon mode and c) radiation study.

The 19th International Workshop on Neutrinos from Accelerators-NUFACT2017

25-30 September, 2017

Uppsala University, Uppsala, Sweden

\section{${ }^{1}$ Speaker}




\section{Introduction}

Experimental Muon Source (EMuS) is a project to be running at the CSNS (Fig. 1). The CSNS is a new facility under construction at IHEP's Dongguan campus [1]. Consisting of an $\mathrm{H}-$ linac and a proton rapid cycling synchrotron, it is designed to accelerate proton beam pulses to $1.6 \mathrm{GeV}$ kinetic energy at $25 \mathrm{~Hz}$ repetition rate, striking a solid metal target to produce spallation neutrons. The accelerator is designed to deliver a beam power of $100 \mathrm{~kW}$, with the capability of upgrading to $500 \mathrm{~kW}$.

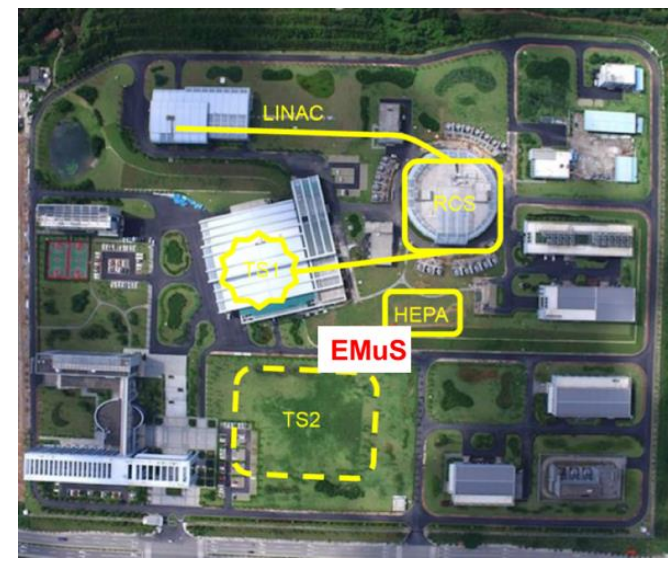

Figure 1 Layout of CSNS

EMuS is located at the HEPA of CSNS (Fig. 2). It will use $4 \mathrm{~kW}$ in CSNS phase one and $20 \mathrm{~kW}$ in phase two of the CSNS proton beam power. The project will run in different modes (e.g. neutrino mode for neutrino physics, surface muon mode for muSR, etc.) for different scientific purposes. Secondary particles are produced at the target and collected efficiently in the solenoids with high magnectic field. High momentum pions are rejected in a selection section. The remaining particles are transported into a $25-\mathrm{m}$ pion decay channel, where muons are produced by the pion decays. For surface muons, they are moderated before transporting to the muSR spectrameter. For decayed muons, they are transported to the decay muon section.

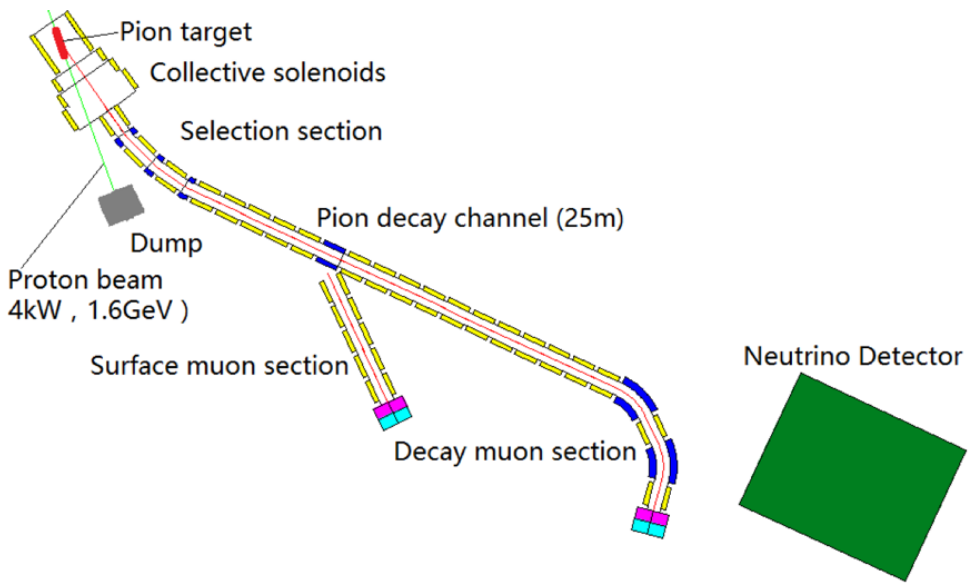

Figure 2 Layout of EMuS 
EMuS is designed to carry out studies such as MOMENT [2] R\&D, muSR applications, neutrino cross section measurement, muon physics, etc. The design and R\&D is supported by a NSFC key instrument development project from 2016/01 to 2020/12. In this presentation, we will report the recent progress in EMuS project. There will be three topics, including a) base design for neutrino mode, b) design optimization for surface muon mode, c) radiation on the solenoids.

\section{Progress in EMuS}

\subsection{Base design for neutrino mode}

In the first section, we report the base design for neutrino mode. The proton beam of $1 \mathrm{~Hz}$ repetition rate is extracted from the RTBT. From MAD-X [3] calculation, the minimal beam size at the target is about $5.2 \mathrm{~mm}$. As the target is placed in the high megnetic field, the proton beam is deflected before it shoots on the target. So two dipoles with $1.37 \mathrm{~T}$ magnetic field are placed before the solenoids to bend the beam by 5 degrees.

The target station consists of 4 superconducting coils with a total length of $2.5 \mathrm{~m}$ (Fig. 3). The first coil's aperture is about $1 \mathrm{~m}$, in which the target is placed. The magnetic field decreases adiabatically from the target to the end of the coils. It is designed to produce a maximum magnetic field of $5 \mathrm{~T}$. The fields are various for differnt running mode. For neutrino mode, we use $(5+3) \mathrm{T}$ magnetic field. The proton beam and the target are tilted by 15 degrees w.r.t. the axle centre of the solenoids. Tungsten shields are placed inside the solenoids for radiation protection.

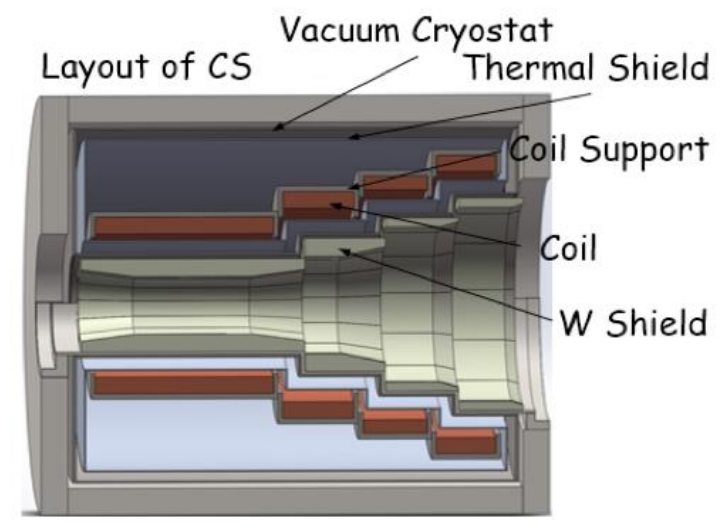

Figure 3 Layout of the target station

The selection section is located $4 \mathrm{~m}$ downstream of the target. The dipole with $1.5 \mathrm{~T}$ field can bend the particles by $30 \mathrm{deg}$ and select particles of interest. The spent protons are collected at the beam dump. As the spent proton in differnt fields distributed in the same polar but differnt azimuth angles, more than one beam dumps will be placed at $8.5 \mathrm{~m}$ downstream of the target.

With the parameters that be presented so far, for neutrino mode, we will have $10^{16}$ neutrinos $/ \mathrm{m}^{2} /$ year at the $3 \mathrm{~m}$ upstream of the decay tunnel assuming a $50 \mathrm{~cm}$ aperture for the first solenoid. The number of neutrinos can be increased by a factor of 5 if we double the aperture. In the base design, the $50 \mathrm{~cm}$ aperture is a trade-off between the performance and the expense. 


\subsection{Design optimization for surface muon mode}

As we have already known, muSR is one of the most important motivations for the EMuS project. In this section, we report works on the design optimization for surface muon mode. The product of muon intensity and squre of polarization $\left(\mathrm{IP}^{2}\right)$ is a common figure of merit (FOM) in the muSR community. We optimize the production target and the magnetic field design by maximize the $\mathrm{IP}^{2}$. For target material, the lower $\mathrm{Z}$ materials, such as $\mathrm{C}$ and $\mathrm{Be}$, have higher pion yields and lower radiation and power densities deposited. For target radius, target with radius between 3 to 4 times of the beam size can produce optimal particle yields. For the angle between the target and the axle centre of the solenoid, the surface muon yields are proportional to this angle. However, the angle is restricted by the aperture of the solenoid. Currently, we use 15-degree in the base design.

The magnetic fields are also optimized for the surface muon operation mode. In principle, lower field produces less contaminated decayed muons, which can improve the muon beam's polarization. We optimize the $\mathrm{IP}^{2}$ by varying the magnetic fields intensities and shapes. Our study shows that the (1+0.5) T magnetic field with fast decreasing rate is optimal for $\operatorname{IP}^{2}$ (Fig. 4).

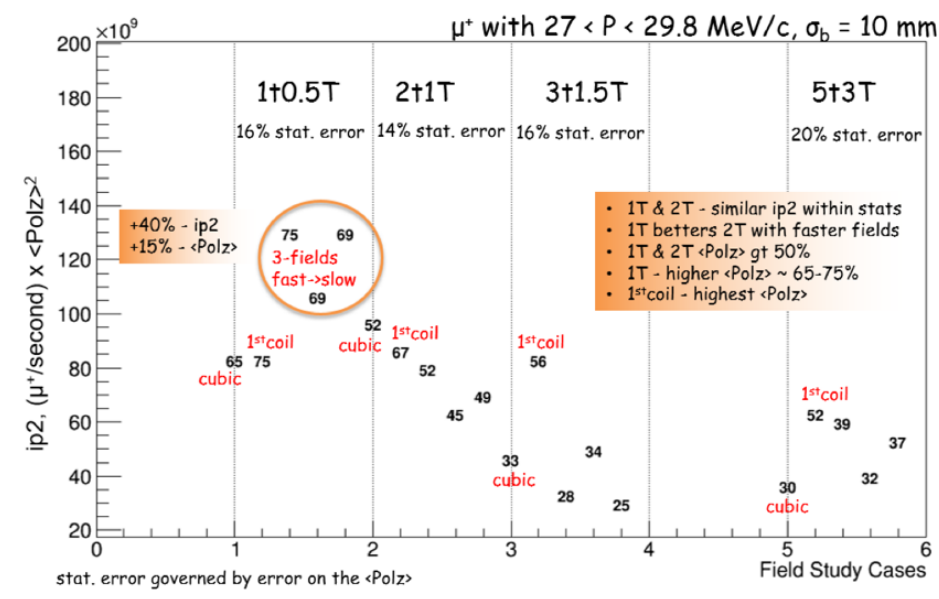

Figure $4 \mathrm{IP}^{2}$ in different magnetic fields

\subsection{Radiation on the solenoids}

In this section, we report the recent FLUKA [4] simulations of radiation for the EMuS target system. The radiation study of the solenoids is crucial for the target station. We have considered three main issues for the radiation:

- Damage to the superconductor's stabilizers

- Maximum local radiation dose to the superconductor insulator over the lifetime of the experiment

- Local heat load allowed anywhere within the superconducting coils

Radiation affects the electrical conductivity of the component metals of the superconductor cable. The Residual Resistivity Ratio (RRR) is defined as the ratio of the electrical resistance at room temperature of a conductor to that at $4.5 \mathrm{~K}$. RRR decreases in the radiation environment. If RRR drops below a certain limit, the stability of the magnet will degrade significantly. In this work, we calculate the RRR for different stabilizers (Figure 5). It turns out that the aluminum stabilizer can survive after 3-month continuous running, while the copper stabilizer can survive 
for one-year operation. On the other hand, RRR can be recovered by warming up the superconducting coils to room temperature. The recovery abilities are different for aluminum and copper. For each thermal cycle, aluminum can be $100 \%$ recovered and copper can only be recovered by about $80 \%[5,6]$, which means the performance of copper will drop dramatically after several thermal cycles. As a result, aluminum is the better material for the coils stabilizer.
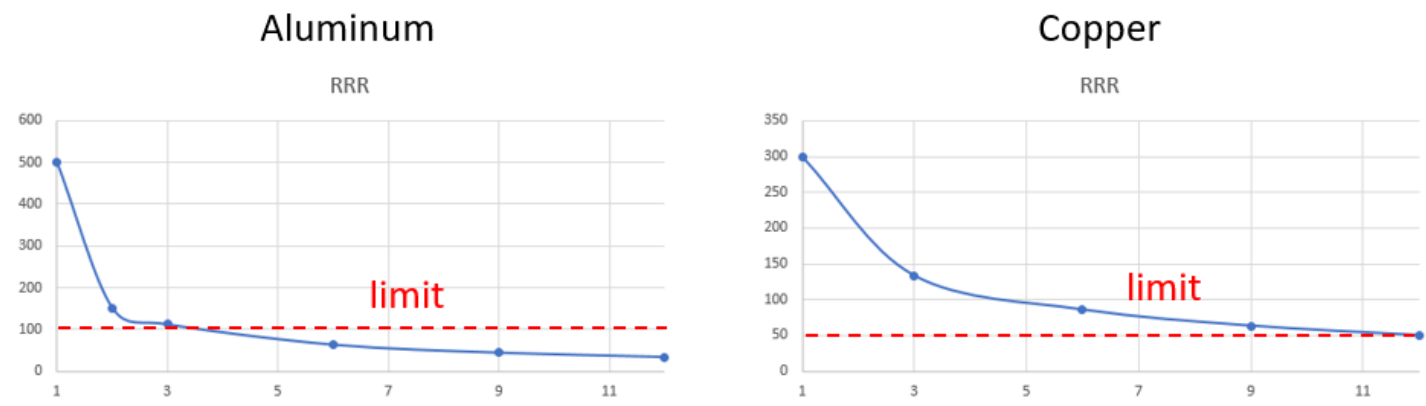

Figure 5 RRR as a function of time (month). The RRR limit for aluminum (copper) is 100 (50).

The mechanical properties of the material can be degraded in radiative environment. The most radiation-sensitive material sets the lower limit for the maximum allowable local radiation dose over the operational lifetime of the EMuS project. In particular, the epoxy used to bond the insulation to the superconducting cable can tolerate a maximum of 7 MGy dose before it experiences a $10 \%$ degradation in its shear modulus [7]. From our simulation, the maximum dose for the insulator in the capture solenoids and the matching solenoids are 1 MGy for one-year operation (Figure 6 in left).
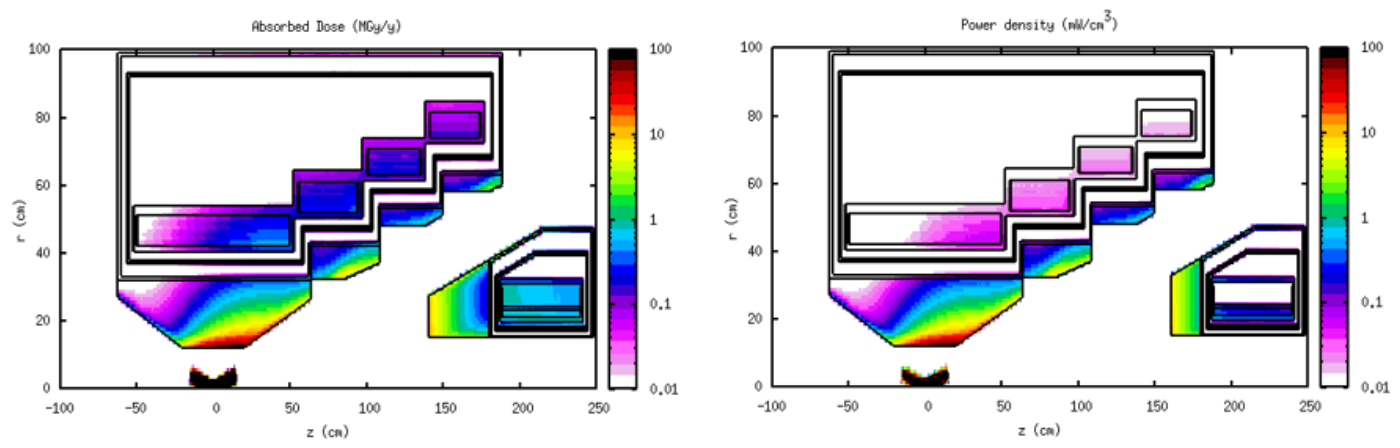

Figure 6 The absorbed dose and power density for the target station.

The maximum power density in the solenoids must meet the requirements that never make the cable turn temperature caused by the deposited energy be above the solenoids critical temperature. From our simulation, the peak power density is $0.15 \mathrm{~mW} / \mathrm{cm}^{3}$ for the capture solenoids and $0.7 \mathrm{~mW} / \mathrm{cm}^{3}$ for the matching solenoids (Figure 6 in right). The values are much below the common limits for the current collider superconducting magnets [8]. 


\section{Summary}

In this talk, the recent progress in EMuS project is reported. A base design is proposed for the neutrino mode, and an optimized design is proposed for the muSR mode. The radiation is also studied in FLUKA.

\section{Acknowledgments}

This work was supported by the National Natural Science Foundation of China (Grant No. 11527811 and 11335009).

\section{References}

[1] Hesheng Chen and Xun-Li Wang, China's first pulsed neutron source, Nature Materials 15, 689691 (2016).

[2] Jun Cao et al. , Muon-decay medium-baseline neutrino beam facility, Phys.Rev.ST Accel.Beams 17 (2014) 090101.

[3] http://mad.web.cern.ch/mad/.

[4] http://www.fluka.org.

[5] J. Horak and T. Blewitt, Isochronal Recovery of Fast Neutron Irradiated Metals, J. Nucl. Mat., 49 (1973/74) 161-180.

[6] M. Guian et al, Defect Production and Recovery in FCC Metals Irradiated at 4.2 K, J. Nucl. Mater. $133 \& 134$ (1985) 357-360.

[7] A. Zeller et al, Radiation Hard Coils, http://supercon.lbl.gov/WAAM.

[8] N. Mokhov, Beam-Related Design Limits for Superconducting Magnets, RESMM'15. 